\title{
¿Qué piensa el alumnado sobre sus profesores de educación física?
}

http://dx.doi.org/10.11606/1807-5509201800040603

\author{
Didier Fernando Gaviria CORTES \\ Francisco Javier Castejón OLIVA ${ }^{* *}$
}

*Instituto Universitario de Educación Física, Universidad de Antioquia, Medellín, AN, Colombia. **Departamento de Educación Física, Deporte y Motricidad Humana, Universidad Autonoma de

Madrid, Madrid, MD,

España.

\section{Resumen}

La investigación sobre el pensamiento del alumno que se presenta en este trabajo, se basa en que el estudiante juega un papel activo y fundamental en su propio proceso de aprendizaje. Este tipo de investigación deduce que lo que los estudiantes crean, piensen y sientan, puede afectar la forma de entender los contenidos que se enseñan y el valor que ellos pueden tener para sus vidas, el gusto por la asignatura y su aprendizaje. En este sentido, el objetivo fue interpretar y analizar las creencias y percepciones que tienen los estudiantes acerca de sus profesores de educación física. La investigación fue cualitativa. El diseño es un estudio de caso donde se ha utilizado cuestionario, grupos focales y observación como estrategias de recolección de información. El estudio se realizó en una institución educativa de Medellín-Colombia. Participaron tres docentes y 103 estudiantes de último grado de educación secundaria. Como conclusión, los estudiantes creen que un buen profesor es aquel que los respeta y trata a todos por igual, sin hacer discriminación alguna, explica bien para qué sirven los contenidos y actividades, cuáles son sus objetivos, es justo en las pruebas y cuando evalúa. Los estudiantes tienen una imagen de los profesores entre dos direcciones: a) el profesor como amigo y guia que aporta elementos positivos para su formación; b) el profesor poco exigente, sin autoridad y falto de liderazgo.

Palabras clave: Estudio de Caso; Pensamiento del Alumno; Profesorado; Proceso de Enseñanza Aprendizaje.

\section{Introducción}

Se ha desacreditado la educación que se reduce al adiestramiento, la memorización y la reproducción cuando debería atender más a la emancipación, la diversidad, la construcción del conocimiento, la ética y la crítica ${ }^{1}$. Debe ser una educación orientada a tener una mente bien ordenada ${ }^{2}$, donde el profesorado actúa como guía cognitivo del alumnado, que le ayuda a apropiarse del conocimiento, para que pueda pensar y aplicar dichos conocimientos por sí mismo. Todo esto exige que la información sea pertinente, que provoque una reorganización acertada y que se pueda comprobar las interrelaciones entre las diversas ideas ${ }^{3-5}$. En este sentido LeE $^{6}$ y WitTrock ${ }^{7}$, recalcan los efectos que produce el profesorado sobre las percepciones, expectativas, atención, motivación, creencias y actitudes del alumnado y establecen una serie de variables mediadoras del proceso de enseñanza y aprendizaje como: metas, esfuerzo realizado por el estudiante, perseverancia en las tareas, actitudes y satisfacción.

Sin embargo, Silverman ${ }^{8}$, Silverman y Manson ${ }^{9}$, y Chatoupis y Vagenas ${ }^{10}$, entre otros, plantean que hay una mayoría de investigaciones centradas en modelos técnicos y cuantitativos donde se comparan métodos de enseñanza, los comportamientos del profesorado y alumnado, y el tiempo asignado a las tareas de los estudiantes, es decir, en conductas observables, pero no así en lo que piensa el alumnado. Dicho de otra manera, muchas investigaciones se han ocupado de inferir lo que piensa el alumnado por sus conductas, pero sólo un número reducido ha explorado e indagado 
dicho proceso por medio de estrategias cualitativas como el grupo focal, la entrevista y la observación, con el fin de comprobar qué piensa el alumnado y cómo entiende el aprendizaje.

Para Torres ${ }^{11}$, solo contemplando lo que acontece en el interior de la escuela con técnicas o estrategias más flexibles y desde marcos que tengan en cuenta lo social, cultural, emocional, y político ${ }^{12}$, es cuando se puede llegar a comprender y, por consiguiente, mejorar la calidad de la enseñanza y los aprendizajes en la escuela.

La investigación desde el pensamiento del alumnado como parte del paradigma de los procesos mediadores, concibe una relación e influencia entre las acciones de los protagonistas del proceso de enseñanza y aprendizaje. Durante el desarrollo de la enseñanza, aquello que los profesores hacen, influye en el comportamiento de los alumnos y viceversa, los comportamientos del alumno, tienen que ver con la forma como los profesores planean y desarrollan la enseñanza ${ }^{3,13}$. Esta reciprocidad supone efectos en las dos direcciones. Si bien la conducta del profesorado ha sido investigada desde diferentes perspectivas ${ }^{14,15}$, el pensamiento del alumnado no ha sido tan prolijo $^{16}$, pues se ha centrado más en su rendimiento sin tener en cuenta sus pensamientos, ideas y creencias. "La investigación sobre los procesos de pensamiento del alumno, se ocupa del modo en que la enseńanza o los docentes influyen en aquello que los alumnos piensan, creen, sienten, dicen o hacen y que tiene repercusión en su rendimiento"'.

Las investigaciones que abordan el pensamiento del alumnado sobre los procesos de aprendizaje, se cuestionan, en cuanto al rendimiento: “¿Cómo aprenden los estudiantes la instrucción que se da en clase? ¿Cuáles son los procesos inmediatos y a medio plazo que la enseńanza genera en los estudiantes?" 17 . Pero también hay otras cuestiones sobre su implicación en las tareas: ¿Cómo el alumno percibe las demandas de las diferentes tareas del aprendizaje escolar? ¿Cómo el alumno se involucra autónomamente en el desarrollo de los procesos que requiere las tareas? ¿Qué tipos de procedimientos mentales utiliza para organizar el conocimiento disponible, asimilar nuevos materiales de contenido y relacionar conceptos para resolver problemas? ${ }^{18}$.

En este sentido, Harnischfeger y Wiley ${ }^{19}$ dicen que los mejores resultados académicos son para aquellos estudiantes que son capaces de generar su propio ambiente de trabajo, desarrollar sus tareas de forma autónoma, sin tener presente en todo momento el comportamiento del profesor o de los materiales utilizados en el proceso educativo. "Es importante dar oportunidades a los alumnos para que usen sus propias experiencias, aunque también es esencial ayudarlos a desarrollar una conceptualización más compleja de lo que se aprende" ${ }^{\text {. }}$.

La educación desde este enfoque supone algo más que el almacenamiento y la recuperación de la información; implica ayudar a los estudiantes en la aplicación de los conocimientos y a pensar por sí mismos; todo esto exige la información pertinente, el estudio de sus interrelaciones y la reorganización acertada por parte del sujeto, donde el profesor actúa como mediador ${ }^{3-5,20}$.

La mediación surge de las tendencias psicológicas y sociales que han sido fuente de importantes trabajos en torno al currículum oculto de la escuela y el aula ${ }^{21,22}$. Principalmente de la psicología cognitiva, que considera que la esencia de todo acto de aprendizaje o resolución de problemas es el rol activo desempeñado por el estudiante al transformar el mensaje (estimulo-respuesta) ${ }^{17}$.

Se puede percibir entonces dos corrientes paralelas de acción, que se presentan entre el profesor y el alumno: a) lo que el alumno entiende del contexto social de la situación en el aula: la manera en que se distribuye los turnos, el carácter de los elogios y los reproches, los estándares implícitos de la actuación, las claves empleadas para señalizar las oportunidades de participar, o los cambios de tareas, etc.; y b) una representación y construcción mental del contenido cognitivo de lo que se está enseñando: conceptos nuevos que se relacionan o comparan con otros aprendidos, estrategias metacognitivas para dirigir y controlar la capacidad intelectual y conocimientos necesarios para comprender mejor los nuevos puntos de vista ${ }^{17}$.

Teniendo en cuenta todo lo anterior, la investigación sobre el pensamiento del alumno se basa en que el estudiante juega un papel activo y fundamental en su propio proceso de aprendizaje ${ }^{23}$; en este sentido nos dice el autor, que la enseñanza, en buena medida, no influye directamente en el rendimiento del estudiante, sino que hace que el alumnado se apropie de las ideas y construye su propio entendimiento que conforma una determinada forma de comportarse. Esta línea de investigación supone que lo que los estudiantes crean, piensen y sientan, puede afectar la forma de entender los temas que se les enseñan y el valor del contenido para sus vidas, pero también cómo se comportan y lo que aprenden en cada una de sus clases.

Para Carreiro da Costa ${ }^{24}$ las referencias conceptuales y metodológicas, en las cuales se sustenta la enseñanza de la educación física, son básicamente: 
el paradigma proceso-producto; el paradigma de los procesos mediacionales del pensamiento del profesor y el paradigma de los procesos mediacionales del alumno (cognitivo y socio-afectivo), cada uno de ellos pone la mirada en diferentes variables y dimensiones de análisis. Cada uno de estos puntos de vista ha proporcionado saberes y reflexiones pedagógicas, con la intención de ayudar a los profesores a organizar y conducir con más rigurosidad científica y mayor conciencia su enseñanza, haciéndolo así aún más eficiente y competente en la enseñanza de la educación física.

Las investigaciones sobre los procesos cognitivos, socio-afectivo y motrices en educación física, se han centrado en tres temas principales ${ }^{16,23,25-28}$ :

Percepciones personales de los estudiantes: los estudios se han centrado en preguntas como: ¿cuáles son las actitudes de los estudiantes hacia la educación física?, ¿cuáles son las expectativas de los estudiantes respecto a la educación física en la escuela?, ¿cuáles son los niveles de satisfacción de los estudiantes al final de las clases de educación física?, ¿qué es una buena lección de educación física para los estudiantes?, ¿¿cuáles son las relaciones entre las características (edad y sexo), la experiencia (éxito o fracaso) y la competencia percibida por los alumnos, orientado a metas, expectativas y valores?, ¿cuáles son las percepciones de los alumnos sobre las finalidades de la educación física?, ¿cuál es el autoconcepto de capacidad de los alumnos en educación física?, ¿qué objetivos persiguen los alumnos en la clase de educación física? ${ }^{29,30}$

Las percepciones de los alumnos sobre el profesor de educación física: se centra esta línea en preguntas como: ¿qué comportamientos de la enseñanza de la educación física valoran más los estudiantes?, ¿cómo interpretar los comportamientos de los profesores?, ¿cómo valoran al profesor? ${ }^{31-33}$

Los procesos cognitivos de los alumnos y el compromiso motor durante las clases: Se hace preguntas como: ¿cuáles son las relaciones entre las características individuales del estudiante, sus creencias sobre educación física y sus comportamientos en las clases de educación física?, ¿las metas que los estudiantes tengan sobre el aprendizaje y sus creencias sobre el éxito y el fracaso en educación física influyen en la forma en que dan sentido a la participación en las clases de educación física?, ¿los estudiantes reciben toda la información trasmitida por el profesor?, ¿la manera como los estudiantes interpretan la instrucción del profesor es influenciada por la estructura de la enseñanza en sí?, ¿cuáles son las variables cognitivas y socio-afectivas de los estudiantes relacionadas con los diferentes patrones de participación en las clases de educación física?, ¿cuáles son las relaciones entre las ideas de los alumnos sobre las condiciones de enseñanza, el proceso de la enseñanza y las características personales (valores, expectativas y motivaciones hacia la educación física) en comparación con su comportamiento en las clases de educación física? ${ }^{34-36}$

Aunque se planteen estos componentes, no se quiere decir que los tres necesariamente se encuentren presentes en todas las investigaciones, pero sí se considera que el componente socio-afectivo es el más importante a la hora de configurar las actitudes que los estudiantes tienen en la clase de educación física y las creencias que tiene hacia la asignatura ${ }^{35,37-39}$.

La percepción del alumnado sobre su desempeño motor, sus objetivos personales en la asignatura, la importancia que la atribuye a la materia y sus aprendizajes en ella, su motivación hacia la práctica, las interacciones que se presentan en el aula, la participación que tienen en la configuración de la asignatura, la preferencia en algunos contenidos, etc., son factores que afectan su actitud en y hacia la educación física ${ }^{40-43}$. La comprensión de las opiniones de los estudiantes sobre cómo experimentan el plan de estudios de educación física, puede dar una idea de cómo ellos le reciben y aprenden.

En esta perspectiva, el estudiante se encuentra influenciado por las condiciones socio-culturales y familiares de donde pertenece, por el proceso de enseñanza y aprendizaje orientado en su institución, como de su propia percepción como alumno. El acceso y la respuesta a la voz del estudiantado son importantes para los investigadores y el profesorado de educación física para desarrollar y elaborar programas de educación física significativos y con un propósito para los propios estudiantes ${ }^{44}$. En este sentido, en este artículo se presenta la información referida al objetivo referido a las creencias y percepciones del alumnado hacia la clase de educación física y su profesorado, como parte de un estudio más amplio acerca del pensamiento del alumnado.

Según Cubero 45 , "los niños y niñas desarrollan creencias y expectativas sobre el mundo natural y social con una gran profusión de términos, que dejan translucir unos determinados compromisos teóricos subyacentes... indica que a lo largo de su experiencia cotidiana los alumnos han desarrollado explicaciones autónomas sobre ellos mismos, los otros y el mundo en general".

Según López ${ }^{46}$, en el contexto educativo las creencias son asociadas erróneamente a juicios, axiomas, percepciones y opiniones. El concepto de creencia se encuentra relacionado con pensar alguna 
cosa que es o puede ser verdad y dice al respecto: "se cree lo que se recibe de nuestros mayores, del ambiente cultural y social y muchas veces, exclusivamente por el mero hecho de que todo el mundo lo dice" ${ }^{36}$. En este sentido, las creencias van a estar presentes en todos y cada uno de los fundamentos que hacen parte del proceso de enseńanza y aprendizaje; creencias acerca de cómo se enseña y cómo se aprende, así como los principios que sustentan las diferentes decisiones sobre la enseñanza, la evaluación, los aprendizajes que se eligen y la comprensión de estos por parte de los estudiantes, las funciones que deben cumplir los docentes y las misiones que la escuela tiene con la sociedad y con cada sujeto ${ }^{47}$.

Parafraseando los planteamientos de Pehkonen y TöRneR ${ }^{48}$ y llevándolos a la educación física, se puede decir que las creencias, pueden tener un poderoso impacto en la forma en que los estudiantes aprenden, utilizan, comprenden y practican la educación física, y por tanto, pueden convertirse en un obstáculo para su aprendizaje. Los estudiantes que tienen unas creencias rígidas y negativas de la educación física, su función y aprendizaje, fácilmente se convertirán en aprendices pasivos y desmotivados hacia la asignatura. Así pues, "la evaluación positiva o negativa de un objeto social dado, o actitud, en la que el sujeto se encuentra implicado, depende de las creencias o convicciones que el sujeto tiene" ${ }^{19}$.

Según GonÇALVES ${ }^{40}$ después de la intervención del profesorado en las clases, los estudiantes pueden efectuar atribuciones de causalidad de acuerdo con varios factores:

a) Factores exteriores: la atribución es relacionada con las características del profesor (es simpático, correcto, si trata diferente a niños y niñas, o a los mejores y más débiles...).

b) Auto-atribución: el alumno basa sus atribuciones causales en hechos personales. Considera una evaluación negativa como justificación de su propio comportamiento o su bajo nivel de desempeño motor.

c) Los factores tienen relación con el ambiente de clase: el momento de la clase, la dificultad de la tarea, más que factores relativos al profesor $\mathrm{y}$ al propio alumno.

d) Una eventual combinación de los tres factores anteriores.

Sin embargo, Gonçalves ${ }^{40}$ plantea que el comportamiento diferenciado o inapropiado del profesorado puede no ser percibido por los estudiantes, no habiendo, por consiguiente, grandes posibilidades del desempeño del estudiante a ser afectado por las expectativas originales del profesor; de allí que posteriores estudios consideren las percepciones de los estudiantes relativas al hipotético comportamiento del profesor.

Las emociones positivas pueden producir comportamientos de ayuda, generosidad y comprensión interpersonal. Transforman a los individuos haciéndolos más saludables, integrándolos a la comunidad, eficientes y resistentes al estrés, siendo factores que con el tiempo producen buenos resultados en la sociedad ${ }^{50,51}$.

El interés por conocer las creencias de los estudiantes acerca de la asignatura de educación física y su profesorado, radica en el hecho de que ellas inciden en sus actitudes, ayudan a explicar y ofrecen líneas para tratar de modificarlas o transformarlas. Las creencias influyen en la forma en que se aprende, se enseña y se práctica la educación física. Aunque en ocasiones es difícil cambiar las creencias y prácticas que se tiene, se pueden intentar reconducir de alguna forma; se puede decir que algunos cambios en las prácticas de la asignatura pueden modificar las creencias tanto del alumnado como del profesorado. Teniendo en cuenta todo lo anterior, el estudio tuvo como objetivo, analizar e interpretar las creencias y percepciones del alumnado hacia la asignatura de educación física y el profesorado.

\section{Método}

El estudio es cualitativo, utilizando estrategias cuantitativas y cualitativas. Se llevó a cabo mediante el diseño de estudio de caso. El estudio de caso no excluye utilizar técnicas cuantitativas, de ahí que asumiéramos esta combinación. Un caso según Coller $^{52}$, Merrian ${ }^{53}$, STAKe $^{54}$, y Yin ${ }^{55}$, es un objeto de estudio con unas fronteras más o menos claras que se analiza en su contexto real y que se considera relevante bien sea para comprobar, ilustrar, o construir un conocimiento o una parte de él, bien sea por su valor intrínseco. Según Galeano ${ }^{56}$ el objetivo del estudio de casos es comprender el significado de una experiencia, e implica el examen intenso y profundo de diversos aspectos de un mismo fenómeno, es decir, 
es un examen de un fenómeno específico, como un programa, un evento, una persona, un proceso, una institución o un grupo social.

Por tanto, la decisión de centrarnos en el estudio de caso radicó en que este diseño se elige precisamente porque el investigador está interesado en el descubrimiento, comprensión y la interpretación más que en la verificación de hipótesis ${ }^{18}$; y resultaba coherente con el tipo de pregunta que deseaba investigar, ya que sus respuestas solo serían posible encontrarlas en el contexto natural, lo cual necesitaba de observaciones y entrevistas durante un periodo escolar.

\section{Contexto y participantes}

La investigación se realizó en una institución educativa pública de la ciudad de MedellínColombia; la cual está compuesta por amplios espacios para la práctica de la educación física y gran variedad de materiales las actividades. Las clases de educación física tienen una duración de 50 minutos. Para el grado observado se orienta la asignatura en un bloque de 100 minutos a la semana. Los participantes fueron 103 estudiantes del último grado de secundaria. La edad de los discentes oscila entre los 16 y 19 ańos de edad. Estos estudiantes se eligieron por su experiencia en el proceso educativo, ya que estaban culminando su formación básica. Son estudiantes con suficiente capacidad de opinión y crítica, comprender las preguntas planteadas y son capaces de expresar sus sentimientos, pensamientos y percepciones sobre la clase de educación física y su profesorado. La institución cuenta con tres profesores para la asignatura de educación física, poseen título de licenciado en educación física y especialización en diferentes temáticas relacionadas con el área. A la fecha tenían entre 11 y 19 años de experiencia como profesores de escuela, además de combinar esta labor en algunas ocasiones con el entrenamiento deportivo en otros espacios de formación.

Con el fin de mantener los criterios éticos de la investigación, se solicitó al centro educativo permiso para realizar la investigación. Se pidió autorización al alumnado, profesorado, dirección del centro y responsables del alumnado (padres y madres). Se expuso en qué consistía el estudio, el anonimato del centro y de todas las personas implicadas. También se expuso que los resultados se entregarían al profesorado y a la dirección del centro educativo. En el mismo documento, se exponía que este estudio se trataría de publicar. Todas las partes estuvieron de acuerdo y dieron su consentimiento.

\section{Las estrategias de obtención de información}

Teniendo presente que la mayoría de los estudios sobre el pensamiento del alumno, se han llevado a cabo por medio de cuestionarios ${ }^{6}$. Nos dice Coller $^{52}$ que, para el análisis de los estudios de caso se pueden utilizar estrategias diferentes, desde entrevistas semiestructuradas hasta análisis de contenidos de documentos, pasando por la encuesta u observación participante. Teniendo presente el objetivo de estudio, el contexto y el marco teórico de la investigación, se optó por unas estrategias para preguntar a la realidad ${ }^{57}$ : el cuestionario y los grupos focales. Y una estrategia para contemplar la realidad ${ }^{57}$ : la observación.

El cuestionario fue la primera estrategia utilizada para la recolección de información, nos permitió valorar la percepción y las creencias desde la realidad de los estudiantes acerca de la asignatura y su profesorado. Además, nos facilitó la organización de los grupos focales y para contrastar los datos con la observación de clases y el grupo focal. Se optó por usar el cuestionario propuesto y validado por GonÇALVES $^{40}$, para luego realizar una traducción, validación semántica y adecuación del lenguaje al contexto de los estudiantes donde fue aplicado. Contuvo 16 preguntas, 13 cerradas y tres abiertas y constituido por ocho partes: caracterización del alumnado, condiciones educativas para la asignatura, grado de satisfacción, actitudes hacia la educación física, percepciones sobre el profesorado, objetivos de la clase, auto-concepto, aprendizajes propiciados en la asignatura.

El grupo focal nos permitió apreciar las percepciones y experiencias de los estudiantes desde su propio discurso, fue un espacio de sentimientos, pensamientos, opiniones, interacción y vivencias sobre la asignatura y el profesorado. El grupo focal en términos de Hamui-Sutton y Varela ${ }^{58}$, es un espacio de opinión para comprender el sentir, pensar y vivir de las personas. Se construyó una guía de entrevista a partir de la revisión de la literatura y el análisis del cuestionario, se realizó una prueba piloto para luego ser aplicada a los estudiantes del estudio. Fueron grupos conformados por seis estudiantes, con el fin de posibilitar una mejor participación y con una duración de 45 minutos en promedio por encuentro.

La observación nos ayudó a identificar las interacciones que se dan en el proceso de la clase entre 
estudiantes-profesorado y estudiantes-estudiantes. Con la observación se buscó recoger fielmente la realidad escolar. En ellas se anotaron las conductas y respuestas a preguntas que aparecen de forma natural. Se observó los grupos durante cuatro meses, en periodos de una hora y cincuenta minutos semanales y el registro se realizó a partir de notas de campo ${ }^{59}$.

\section{Tratamiento de la información y momentos del estudio}

El análisis y tratamiento de la información se realizó en tres fases consecutivas así planteadas por TAYlor y BOgDAN ${ }^{59}$ : de descubrimiento, de decodificación y de relativización. Con el cuestionario se realizó un análisis descriptivo mediante el programa SPSS versión 20, y se trató las frecuencias y porcentajes de las respuestas del alumnado con el fin de que nos sirviera para obtener información a comprobar en los grupos focales y la observación. En cuanto a las observaciones y los grupos focales, se aplicó el software para el análisis de datos cualitativos Nvivo 10 versión 10.0.3 para Mac.

Teniendo en cuenta el tratamiento anterior, en el presente artículo se identifica los cuestionarios con el código CE (cuestionario estudiantes), las observaciones OC (observación de clase) y los grupos focales GF. Cada uno de estos códigos estará acompañado de un número (CE1, OC1, GF1), el cual corresponde a un estudiante participante, de esta forma se puede mantener el anonimato.

\section{Resultados}

Percepción general del alumnado sobre sus profesores. Se les preguntó a los estudiantes su opinión sobre cuáles son las cualidades de un buen profesor de educación física. Se les pidió seleccionar las cinco cualidades más importantes para ellos de un buen profesor. En este sentido, las cualidades más valoradas por los estudiantes de grado once fueron las siguientes:

Cualidad más importante:

"Respeta a los alumnos y los trata de forma correcta".

Segunda cualidad más importante:

"Explica bien para qué sirven los ejercicios, cuáles son sus objetivos y cómo deben de ser hechos".

Tercera cualidad más importante:

"Es justo en las pruebas y cuando nos evalúa".

Cuarta cualidad más importante:

"Establece junto con los alumnos reglas de comportamiento, lo que los alumnos y el profesor deben de respetar".

Es importante resaltar, que la primera cualidad en importancia (respeto a los estudiantes) fue la más nombrada por los estudiantes del estudio $(78 \%)$, ya que los que no la pusieron de primera, la nombran de segunda o tercera en importancia. Es decir, el respeto y la forma de dirigirse a ellos como estudiantes, es fundamental en una buena relación y en la percepción del estudiantado hacia el profesorado y la clase de educación física. Según NolAsCO $^{60}$, aquello que el estudiante acredite en el perfil de un buen profesor, gana importancia en la medida en que la comparación de sus creencias con las características observadas en el profesor, puede conducir a diferentes actitudes hacia el profesorado, influenciando la interacción pedagógica en la clase de educación física.

Otros comentarios hechos por los estudiantes acerca de las cualidades importantes en el profesorado fueron: M1: Pues yo digo que él también debería interactuar.

H2: Que interactúe con los estudiantes.

M2: Pues porque nosotros como estudiantes siempre queremos que nos estén motivando y no es solamente que nos digan hagan esto y esto [...] uno siempre tiene que tener un profesor que lo esté motivando. GF.1.

M1: Comprensión.

H1: Sí.

H3: Sí más que todo eso, saber tratarlo a uno, porque si un profesor no sabe tratarlo a uno, uno qué va estar haciendo ahí.

M2: Y también que se le vea como esas ganas de enseñar, pues que se note que le guste enseñar, no que llegue como obligación, [...] pues los profesores son el reflejo de lo que uno va aprender por decirlo así y por ejemplo, si llega aburrido a dar clase a uno no le van a dar ganas de aprender, si llega motivado, no muchachos vamos aprender una cosa buena, entonces hay si me gusta [Risas]. GF.3.

H1: Pues yo me imagino el profesor de educación física tiene que saber pues. 
H2: Y que sea bien especializado en lo que va hacer, y responsabilidad ante la clase.

D: Que conozca bien del tema.

M1: Sí.

H2: Y responsabilidad ante la clase.

H1: Que prepare la actividad.

H2: Eso que prepare clases.

$\mathrm{H} 2$ : Que esté pendiente siempre, ah, que vio que tal persona está haciendo algo mal, acercársele y decirle a vea compañero en tal cosa estaba fallando, esto y esto. GF.4.

En este grupo de comentarios que se ha expuesto y que proceden del grupo focal, el alumnado demanda un conocimiento del contenido, en el sentido de que debe dominar la materia que expone, tal y como se solicita en las investigaciones ${ }^{31,61,62}$.

H1: [...] es genial porque él lo hacía con uno, él le mostraba a uno, entonces él bailaba y él hacía todo eso, él hacía el ejercicio con uno, muchas veces jugábamos así, que el pañuelito, que el balón y él jugaba. GF.1.

H1: Un trabajo también recíproco entre estudiante y maestro, entonces que el maestro proponga lo que le toca.

$\mathrm{H} 2$ : Y que se integre también.

M1: Y porque es que lo ponen a uno hacer tal cosa y ya el profesor sale y se va, entonces como el profesor se fue ya uno no sigue haciendo nada. H3: No descuidando la clase.

$\mathrm{H} 2$ : Tiene que tener autoridad.

H1: Lo primero es que esté presente.

M1: Eso.

H1: La presencia es importante.

H3: Demasiado.

H1: La presencia es muy importante porque entonces.

H2: Y que actúe si alguna cosa.

$\mathrm{H} 1$ : Nos pone hacer cualquier actividad y se va hacer otra cosa.

H2: Ajá.

M1: Entonces ya uno no hace nada.

H1: Se relaja uno, uno también se relaja, no que esté presenté, la presencia es muy importante, pues yo creo que eso es uno de los métodos, para que pues, para mantener ahí. GF.4.

$\mathrm{Y}$ esto es lo que perciben en el estudio los estudiantes, cuando consideran que la interacción con el profesorado, su comportamiento, la actitud que el demuestre en la clase y el conocimiento de la asignatura influyen de forma importante en el aprendizaje, el gusto y la actitud de ellos hacia la asignatura.
H1: él tiene que estar para que guíe, pues él no lo puede obligar a usted, pero sí tiene que ser la guía de todos, entonces el aprendizaje depende mucho de él porque entonces... digamos que por una consulta o algo tin [sic] la hacemos, a hacemos pero necesitamos.

H3: Que él nos explique.

H1: Explique el porqué de las cosas.

H3: Para aprender más de la carrera y todo eso. H2: No como por salirse del paso. GF.4.

M2: sí influye, porque si usted entra desmoralizado y hay un profesor como.

H2: Esta ahí, pero no sabe nada.

M2: $\mathrm{O}$ un profesor que no le gusta ni siquiera lo que está haciendo, entonces como que no, como que ¡ah! bueno, pero si uno ve que es alguien como que bien, con moral, que uno muchas veces ni siquiera lo hace por uno mismo, yo tengo que hacer tal actividad, pero si yo sé que la voy hacer y que mi profesor me cae bien y que la voy hacer por sentirlo bien a él, yo lo hago, pero si es un profesor que no.

H3: Por el respeto a él. GF.5.

Ahora bien, al preguntarle al estudiantado qué se les viene a la cabeza cuando escuchan decir profesor de educación física; su concepto del profesorado de educación física abarca o toca algunos de los aspectos mencionados hasta aquí por ellos y los estudios sobre el tema ${ }^{26,40,60}$.

H1: voy a hablar primero a nivel general, sin hablar mal de un profesor, yo diría que depende del profesor, porque hay algunos profesores que son estudiantes y son compañeros de uno, pero hay otros profesores que simplemente es una persona que están al frente dándole instrucciones a uno.

H3: Yo pienso como el compañero, que es apoyando lo que él dice, pues que es el profe Juan, yo pienso que a mí me dicen educador de física, y a mí se me viene a la imagen él y a mí me dicen quién es el para mí, y yo digo que él en mi vida [...] él me ha marcado.

H1: Yo diría que personas así son las que realmente deberían ser llamados profesor. GF.1. M4: sí los profesores de educación física son muy buenas personas, pues no son así [...] que tengamos que decir, nooo qué pereza, esas cosas, no, eso no. GF.6.

Por otro lado, hay quienes no tienen esta imagen del profesorado de educación física y los consideran poco exigentes, con pocos conocimientos, falto de liderazgo en particular 
cuando los llegan a comparar con el profesorado de las otras asignaturas escolares.

M3: Porque si él desde la primera clase él no exige el horario, entonces los demás van a empezar a llegar todos los días tarde.

H3: A hacer lo que se les dé la gana.

H1: sí lo mismo que dijo ella, que pues, por ejemplo, si un profesor le dice usted me va a llegar tarde, si usted me va a llegar tarde lo dejo afuera, entonces usted ya tiene que estar más responsable con eso. GF.2.

M: Relajado, si eso.

H3: Normalmente las otras materias son como más [...] ¡ cómo se dice?, son como las importantes en cambio educación física uno como que ahhh. GF.5.

Como se puede ver, la imagen del profesorado en los datos encontrados se muestra un poco dividida, los que consideran al profesorado de educación física un amigo, un orientador y una persona que aporta a su formación, y los que los consideran poco exigente, sin autoridad y faltos de liderazgo; resultados hallados también por Murcia y Jaramillo ${ }^{63}$ y que pueden deberse, tal y como comentamos antes, al rol de menor estima que desempeña la asignatura para algunos estudiantes ${ }^{64,65}$.

Los estudiantes respondieron a la pregunta del cuestionario ¿demuestra entusiasmo el profesor en sus clases?, un 79,6\% de los estudiantes dice que el profesorado siempre o muchas veces es entusiasta en las clases, solo a veces $18,3 \%$ y pocas veces o nunca $2,2 \%$. Lo anterior demuestra una buena disposición por parte del profesorado a la hora de orientar la sesión de clase. En este sentido algunos testimonios de los estudiantes fueron:

Le da ánimo. CE.1.3.

Su responsabilidad y su actitud con la que da la clase. CE.3.19.

La actitud que tiene en la clase. CE.3.35.

$\mathrm{Su}$ actitud en las clases, transmite ánimo de hacer bien las cosas. CE.3.10.

Estos comentarios hacen que el alumnado sienta que el profesorado les alienta para el desarrollo de las actividades, y sienten y perciben que ayuda a mejorar sus aprendizajes. Se puede decir que despertar el interés de los estudiantes hacia la asignatura de educación física, requiere de un profesorado entusiasmado, que viva y sienta la asignatura; así se transmitirá ese entusiasmo a los estudiantes y el deseo de aprender en la asignatura.

Percepción del trato que recibe el alumnado del profesorado. Frente a los hallazgos en el cuestionario relacionados con los elogios de los profesores hacia los estudiantes en la clase, los estudiantes informan que un $55,9 \%$ solo a veces y/o pocas veces perciben estos elogios por parte del profesor; un 32,3\% perciben que siempre o muchas veces esos elogios; en cuanto el 11,8\% dicen nunca o casi nunca se sienten elogiados en la clase. Sin embargo, en algunas de las clases observadas se pudieron detectar momentos como el siguiente:

el profesor llama a las mujeres y les pide realizar el ejercicio, ellas lo hacen muy bien, lo cual lo celebran todos los estudiantes y el profesor, ellas salen contentas de la actividad porque todos las aplauden y hacen ruido. OC.9.

El profesor se acerca y las motiva para que se acerquen y motiven a jugar; tres mujeres se paran y les dicen a las otras "vamos" "vamos" las otras entonces se van acercando donde están escogiendo a los integrantes de los equipos. OC.13.

Con relación a la participación de los estudiantes como ejemplo de las actividades y ejercicios a realizar durante la clase a solicitud del profesorado, el 55,9\% perciben que "siempre" o muchas veces" el profesor les solicita demostrar frente al grupo de clase; en cuanto un $31,2 \%$ dice a veces; $12,9 \%$ percibe "pocas veces" o "nunca o casi nunca". Un aspecto positivo encontrado en el estudio, es el hecho que el estudiantado se sienta acogido por su profesorado para ponerlo de ejemplo en el desarrollo de las actividades durante la clase. Este es un punto que puede mejorar el interés y la motivación por la asignatura como se ha tratado en el análisis de tareas ${ }^{15,66-68}$; lo contrario sería que el estudiante se sienta relegado, lejano o rechazado en su participación durante la clase.

El alumnado percibe que el profesorado intenta ofrecer al estudiantado una clase con las mismas condiciones para todas las personas durante la interacción. Se percibe que el profesorado los trata de igual forma a todos, es decir, dispensa la misma atención, actitud y comportamiento con todo el alumnado. La observación anterior nos muestra lo importante y fundamental que puede llegar a ser un elogio para los estudiantes; en estos testimonios, el elogio puede aumentar la confianza en sí mismo, en este caso de las estudiantes. Con una felicitación o aprobación se pueden fortalecer lazos de amistad y solidaridad entre las personas que comparten en la clase de educación física, llegando, por qué no, a mejorar las relaciones entre profesorado y estudiantes. El elogio puede generar satisfacción por lo realizado, motivar para seguirlo realizando y participar con más frecuencia 
de las actividades de la clase, como ya se había registrado en estudios anteriores ${ }^{69,70}$.

La ayuda que el profesorado le presta a los estudiantes durante la clase es fundamental para que el alumnado se sienta acogido y sienta la preocupación del profesor durante el proceso de formación ${ }^{60}$. En este sentido, el 83,9\% de los estudiantes encuestados en el estudio, dicen sentir "siempre" o "muchas veces" la ayuda del profesor cuando tienen dificultades en las tareas de la clase; $14 \%$ consideran que "a veces", y solo el 2,2\% perciben que "pocas veces" son ayudados por el profesor.

$\mathrm{H} 2$ : $\mathrm{Si}$, pues le colaboran en lo que no es capaz.

H3: Por ejemplo, cuando uno no es capaz de hacer un estira.

M3: Aha [...], estiramiento.

H3: Estiramiento.

H2: Sí, o lo está haciendo mal.

M1: O el manejo del balón, la posición del balón por ejemplo en el voleibol, que hay unas personas que juegan así y también hay personas que juegan así trate de.

H2: No pegue así.

M1: Péguele así, no le pegue acá, no pegue así. GF.2.

No nos regaña, nos da consejos para que hagamos tareas que nos benefician, explica con paciencia, es buen profesor. CE.3.8.

En esta secuencia de aportaciones se comprueba la necesidad del apoyo del docente y cómo les afecta en su interés por el aprendizaje. Las percepciones de los estudiantes demuestran la actitud positiva del profesorado para ayudarlos en la clase, cuando ellos encuentran dificultades para llevar a cabo una tarea, como se ha demostrado en el uso del feedback ${ }^{71}$. De igual forma demuestra el interés del profesorado hacia el aprendizaje del estudiante.

La mayoría de los estudiantes (76,3\%) perciben que el profesorado los trata de igual forma a todos, es decir, dispensa la misma atención, actitud y comportamiento con todo el estudiantado. Esto nos lleva a creer que el profesorado logra ser imparcial y equitativo a la hora de prestar atención y cuidado tanto a hombres y mujeres en la clase. Esta percepción de los estudiantes se ve reflejada en algunos de sus testimonios:

El profesor se mantienen al tanto de lo que hacen todos en la clase. CE.1.12.

El profesor tiene respeto hacia todos los alumnos y mantiene la igualdad con todos los compañeros. CE.1.20.
El profesor se preocupa porque todos trabajemos.

CE.2.2.

Como señala el alumnado, esta actitud o comportamiento por parte del profesorado, es un factor favorecedor de actitudes positivas en el estudiantado; ya que según Griffin ${ }^{72}$, los profesionales del ámbito de la educación física, no siempre tienen en consideración este aspecto en el desarrollo de sus clases.

Con respecto a si el profesorado es educado con los estudiantes; los estudiantes (89,2\%) perciben al profesor "siempre" o "muchas veces" ser educado con ellos; $6,5 \%$ dice que solo "a veces", y 4,3\% "pocas veces" o nunca o casi nunca” sienten que el profesor sea educado con los estudiantes. Esta forma cortés del profesorado tratar al alumnado, se ve en el respeto que tienen ellos hacia el estudiante, en este sentido el 93,5\% del estudiantado dicen sentirse tratados con respeto "siempre" o "muchas veces" por parte del profesorado; en cuanto $4,3 \%$ de los estudiantes mencionan que "a veces" se sienten respetados y $2,2 \%$ se sienten irrespetados por el profesorado de la institución educativa. En este sentido, el estudiantado valora algunos comportamientos del profesorado diciendo que le gusta del profesor los siguientes aspectos:

Que es respetuoso, comprensivo y sobre todo que sabe afrontar todo lo referente a la clase. CE.2.20.

El buen trato que maneja con todos sus alumnos y la consideración hacia las mujeres. CE.2.28. La forma en que se puede relacionar como docente-estudiante y el conocimiento que me brinda para formarme como ser. CE.3.33.

el trato que nos da y además su forma de enseña. CE.2.23.

Las aportaciones del alumnado llevan a pensar que en la asignatura de educación física, se respira empatía y unas buenas relaciones entre profesor-estudiante y estudiante-estudiante, lo cual es un factor determinante para el aprendizaje de actitudes positivas hacia la asignatura, con un discurso de los docentes para que se sientan seguros, bien tratados y dirigiendo la atención hacia el aprendizaje, como se ha mostrado en estudios al respecto ${ }^{73,74}$. Igualmente resaltan la importancia del respeto mutuo y el buen trato entre profesor-estudiante; ya que es en el aula donde se dan los aprendizajes. Lograr que el estudiante se sienta respetado, requiere de un gran esfuerzo por parte del profesor, así como de los estudiantes. 
En algunas clases se observó en el profesorado los comportamientos mencionados por los estudiantes, además de algunos comentarios en los cuestionarios.

El profesor los motiva diciéndoles que sí saben bailar, que lo que pasaba era la timidez, que lo hacen muy bien y los alumnos se ríen y aplauden. OC.5.

El profesor se acerca y las motiva para que se acerquen y motiven a jugar; tres mujeres se paran y le dicen a las otras "vamos" "vamos" las otras entonces se van acercando donde están escogiendo a los integrantes de los equipos. OC.13.

En el trascurso del camino el profesor saluda y abraza a varios estudiantes y va conversando con ellos de temas diferentes a la clase. OC.1.

"Que es chévere y charlamos de lo que a pasado". CE.3.31

“...respetuoso y cómico". CE.99.99.

Los hallazgos llevan a pensar que, en la asignatura de educación física dentro de la institución, se respira unas buenas relaciones entre profesor-estudiante y estudiante-estudiante.

Percepción sobre acceso al profesorado. Teniendo en cuenta lo anterior y con el fin que los estudiantes alcancen un mejor aprendizaje y participación en la asignatura, el profesorado debe de crear o seguir propiciando espacios y dinámicas que favorezcan la interacción con sus estudiantes dentro del colegio. Donde las relaciones no solo se basen en aspectos escolares, sino que se puedan establecer algunos aspectos socio-afectivos de ambas partes como sujetos. Se puede señalar algunos de los comentarios que los estudiantes destacan en el profesorado.

Que se puede charlar sin que pierda su posición de profesor. CE.2.25.

Es asequible. CE.2.11.

Que nos enseña muchas cosas, nos trata bien, y además es muy divertido con nosotros. CE.2.21. M2: a mí me parece que él también ha sido buen profesor porque él siempre nos está motivando a todo, por ejemplo, estos días, en estos días nos decía, que como él estaba de coordinador y todo eso nos decía que muchos íbamos muy mal, que nos pusiéramos las pilas, que vea que todavía teníamos pues oportunidades de ganar el año. GF.1.

De estas percepciones se muestra que la intención es interaccionar con los estudiantes de forma que se les permita expresar de forma libre sus opiniones, sentimientos, percepciones y creencias; que perciban al profesorado como una persona que se preocupa y está atenta a lo que ellos sienten, necesitan, piensan y desean.
Disciplina y manejo de conductas disruptivas. Por otra parte, se les preguntó a los estudiantes sobre la disciplina en la clase, específicamente si el profesor les llama la atención cuando no se comportan bien en la clase de educación física. Un 65,6\% de los estudiantes perciben que "siempre" o "muchas veces" el profesor les llama la atención cuando están realizando algo diferente a las tareas orientadas en la clase; para 19,4\% solo "a veces" se presenta esto en la clase; y para el $15 \%$ eso ocurre "pocas veces" o "casi nunca".

muchos continúan sentados conversando o sin hacer nada en las sillas del salón, el profesor les recuerda la práctica que deben de hacer, ellos siguen allí sentados. OC.11.

El profesor ya faltando poco para terminar la clase, le pide a algunos estudiantes que están jugando o escuchando música en sus móviles que los guarden, llega donde los de la guitarra y les pide que guarden inmediatamente esa guitarra, los estudiantes la guardan y se van para una mesa a jugar tenis. OC.12.

El profesor los saluda y les hace un llamado de atención por la hora de llegada y la disposición hacia la clase. OC.2.

De estos datos se comprueba una percepción del alumnado sobre la preocupación del profesorado por mantener la disciplina en la clase y que el estudiante tenga un comportamiento adecuado durante la sesión de educación física, con ello se persigue un clima de armonía y confianza que mejora el proceso de enseñanza aprendizaje, algo ya demandado en los estudios sobre estos aspectos ${ }^{69}$. Sin embargo, algunos estudiantes plantean que muchos de sus compañeros y compañeras de clase no respetan al profesorado de educación física y dijeron lo siguiente al respecto.

M1: allá nosotros practicamos fútbol, pero hay algunos que sabotean, le dicen groserías a Juan, que hijueputa [sic] [...] yo no voy hacer esto, ustedes han visto también y qué piensa usted, a ver a este profesor no lo respetan. Como así, como [...]. H3: Eso va más en el profesor, pues no se hace. M1: Respetar [...] si, no pone seria la clase. GF.2. $\mathrm{H} 1$ : por ejemplo ante todo, si usted no le gusta algo, usted de todas formas tiene que colaborar con el profesor y mostrarle la disciplina pues buena y ya, pues si, y maluco por ejemplo usted, el profesor explicando y usted no le gusta, entonces usted va hacer indisciplina y yo diría que por ejemplo eso aquí si se da, que todos por ejemplo hay mucho que no les gusta pero respetan, respetan lo del otro. GF.2. 
H3: Obviamente si el no mantiene la disciplina entonces que; todo el mundo se revolotea por ahí hacer nada. GF.3.

Como se puede evidenciar, los estudiantes se exigen a ellos mismos un respeto, incluso reclaman al profesorado mucha más actitud para mantener el orden y la disciplina en la clase, hasta se podría decir que el profesorado sea mucho más rígido o estricto al respecto; además, plantean que, las actitudes de los estudiantes tienen que ver o están relacionadas con el comportamiento del profesorado en este aspecto disciplinario.

No obstante, estas actitudes negativas del alumnado hacia el profesorado de educación física y hacia la asignatura, no son las mismas por parte de ellos en otras asignaturas escolares como ciencias sociales, castellano e inglés. En estas asignaturas, en la mayoría de casos, el alumnado se muestra más atento a las explicaciones del profesorado, llegan puntuales a la clase, respetan en general las normas básicas de comportamiento dentro del aula, no se dispersan del trabajo asignado por el profesorado, entre otras actitudes que son diferentes en la clase de educación física, en este sentido se observó lo siguiente:

los estudiantes sacan sus cuadernos y van organizando su puesto de trabajo. Todos los estudiantes ponen atención y escuchan en silencio al profesor. OC.10.

El profesor sale un momento del aula y los estudiantes siguen trabajando en silencio y de forma autónoma. OC.10.

Se siente de repente un silencio en el aula, todos están trabajando en las preguntas, escuchando la lectura y escribiendo; se ven más concentradas en la actividad a los 2 grupos de las mujeres, ellas están en la parte de adelante del grupo y trabajando en mucho silencio. OC.6.

mientras tanto cuatro alumnos apenas llegan a la clase, saludan al profesor, toman asiento y comienzan a trabajar en la prueba. OC.8.

De estos datos se comprueba que hay que tener en cuenta que en algunos rasgos, la asignatura de educación física no siempre es tratada de igual con otras materias, tal y como se ha mostrado en los estudios al respecto ${ }^{64,65}$. Para algunos estudiantes frente a estas actitudes o comportamientos de ellos en las clases de educación física, plantean que se debe en ocasiones al espacio donde se orienta la clase, la actitud del profesorado hacia ellos y el interés del alumnado hacia la asignatura.

M2: Pues lo que dice Ramón eso es verdad, y también se debe al espacio, porque es muy diferente aquí que nos pongan a decir algo, sabiendo que están los balones que ellos llevan, todos cogen los balones y se ponen a jugar y en cambio en un aula uno está sentado y ya el profesor al frente, y es lo mismo que dice él, pues si ellos a veces están hablando entonces uno que hace, uno se pone hablar y el ruido lo dispersa a uno. GF.1.

Percepción del alumnado sobre los conocimientos del profesorado. En otro orden de ideas, se cuestionó a los estudiantes cómo perciben el dominio que tiene el profesorado de los contenidos de la asignatura. Un 86\% aprecia que "siempre" o "muchas veces" el profesor tiene dominio de los contenidos de la clase; en cuanto que para un 9,7\% "a veces" tiene dominio; y para el 3,2\% pocas veces el profesor conoce y domina los que enseña en la clase. Un ejemplo de ello es el siguiente testimonio.

M1: porque así no sepan mucho de un tema, siguen estudiando y consultando para uno, pues para darnos como buena explicación de ellos, sí, y que nos guste. GF.3.

En este sentido, entre los aspectos que más les gustan a los estudiantes del profesor, se encuentra el manejo y el conocimiento de los contenidos de la asignatura, es así que dijeron:

"Que sabe lo que hace y ama lo que hace". CE.1.23.

"Que sabe bien lo que hace, domina el tema y sabe qué hacer en el momento justo". CE.1.28.

"Sabe de lo que habla y así aprendemos los contenidos correctamente". CE.2.17.

\section{Discusión}

La imagen del profesorado se mueve entre los que consideran al profesorado de educación física un amigo, un orientador y una persona que aporta a su formación, y los que los consideran poco exigente, sin autoridad y faltos de liderazgo. De esta forma, los estudiantes perciben a un profesorado que les permite experimentar en el conocimiento, los escucha y tienen una relación horizontal estudianteprofesorado. Y otro profesorado sin credibilidad, conocimientos y reconocimiento institucional. 
De los resultados encontrados, se puede evidenciar que para los estudiantes es fundamental un profesorado de educación física que se integre con ellos, lo cual motiva a los estudiantes a participar con más ganas en las actividades de clase; un profesorado que tenga conocimientos de lo que enseña, esto le da autoridad al profesorado y facilita la disciplina dentro de la clase según se deriva de sus comentarios; un profesorado que los respete y trate bien, con vocación, liderazgo e innovador; un profesorado que prepare bien las clases y sea imparcial, equitativo y justo a la hora de evaluar sus procesos en la asignatura; un profesorado abierto, creativo y flexible con la asignatura y que esté presente siempre en clase con los estudiantes, algo que se puede comprobar en la misma línea con los estudios sobre el conocimiento necesario del profesorado ${ }^{75-77}$.

En este sentido dice GALEANO ${ }^{78}$, un educador será lo contrario al maestro pasivo, oculto, encerrado en su cubículo, en su disciplina, en su monólogo tecnocrático; será potencia, energía, vida palpitante, alegría, entusiasmo, algo así como eterna juventud, eterno movimiento intelectual, despliegue de fuerzas, sediento de conocimiento: un investigador y revelador de las leyes de la vida y de su profesión. De modo similar, HernándeZ y LóPEz ${ }^{79}$ concluyeron en su trabajo que la seguridad y la preparación que muestra el profesorado, la capacidad de diálogo, la simpatía y la afectuosidad influyen de forma positiva en el alumnado; y rasgos autoritarios, de antipatía y ser distante con los estudiantes, influyen negativamente en el alumnado.

Un profesorado con la capacidad de integrarse con los estudiantes y los tenga en cuenta en el proceso, posibilitará al estudiante a aventurarse en el mundo del conocimiento, como también a su propia vida, a la vez a formar seres humanos con una dimensión ética, dispuestos a escuchar al otro en una comunicación horizontal, de iguales. Puede enseñarle al estudiante, niño o adulto, rico o pobre, hombre o mujer, que él o ella pueden aprender si se dedican a trabajar en ellos mismos ${ }^{6,16}$. Y un profesorado que no posibilita la participación de los estudiantes, impide al sujeto la formación de todas sus potencialidades, además de generar actitudes y creencias negativas hacia la educación, el profesorado, la escuela y la asignatura ${ }^{31}$.

Muchas veces como profesores, no somos conscientes de la importancia de mostrar o transmitir entusiasmo en lo que hacemos cotidianamente en las aulas y solo nos dedicamos a llenar a los estudiantes de discursos vacíos y sin contexto, que poco sirven a su formación como sujetos y terminamos alejándolos de la asignatura y de nosotros mismos ${ }^{80,81}$.

En pocas palabras, el analizar y comprender las creencias y percepciones generales que tienen los estudiantes sobre la práctica pedagógica que lleva a cabo su profesorado en las clases de educación física, sus interacciones con el profesorado y la competencia de los mismos, puede ser fundamental para entender mejor a los estudiantes y mejorar la práctica docente en la escuela. Según Graham ${ }^{82}$ los mejores maestros son aquellos que se preocupan por entender a sus estudiantes, estos tienen la habilidad de ponerse en los zapatos de los estudiantes y crear e implementar experiencias que son verdaderamente interesantes y beneficiosas para sus estudiantes.

La ayuda del profesorado al estudiante durante la clase, tiene un efecto positivo sobre la motivación y participación por aprender, además ayuda a que el estudiante se sienta aceptado por el profesorado y por la clase. Este punto puede ser una buena estrategia para incentivar al estudiantado a participar en las prácticas de educación física. Según TAPIA ${ }^{83}$, una de las variables que ha sido positivamente valorada por los estudiantes en las clases es la ayuda del profesorado dentro y fuera de clase.

La interacción entre hombres y mujeres como propuesta metodológica en la asignatura de educación física, se convierte en un aspecto fundamental para la transformación de algunos estereotipos acerca de la práctica de la educación física, la actividad física y el deporte de las mujeres; de allí que el profesorado entienda y comprenda la importancia de este aspecto para motivar e incentivar la práctica entre chicos y chicas en las mismas actividades corporales, convirtiendo el espacio de la asignatura en un espacios de convivencia, trabajo en equipo, tolerancia, solidaridad y construcción social en la escuela. En suma, el profesorado del colegio debe de reflexionar acerca de su labor en el desarrollo de conocimientos, valores y actitudes que ayuden a disminuir la discriminación de género en sus prácticas. Identificar y conocer aquellos aspectos que son propensos a generar o desencadenar actitudes y percepciones de discriminación y poca participación de las mujeres en la clase de educación física, ayudará a pensar posibles soluciones, propuestas y proyectos para la institución.

Igualmente se debe de resaltar la importancia del respeto mutuo y el buen trato entre profesorestudiante; ya que es en el aula donde se dan los aprendizajes, lograr que el estudiante se sienta respetado, requiere de un gran esfuerzo por parte 
del profesor, así como de los estudiantes. El fomentar relaciones afectuosas con los estudiantes, es un factor que puede ayudar a mejorar la participación de los estudiantes en la asignatura, las relaciones entre estudiantes y profesorado.

En este sentido, ya lo planteaba Siedentop ${ }^{69}$, que la vida del profesor puede ser más agradable y satisfactoria si se establecen buenas relaciones con los estudiantes; por eso propone unas habilidades de interacción entre profesor y estudiantes entre las cuales se destacan: ser constante en las interacciones con sus estudiantes, tener presente los intereses y necesidades de los estudiantes, hablar con los estudiantes de temas no relacionados con la escuela, mantener un entusiasmo constante durante el proceso de la asignatura, no ignorar los sentimientos y emociones de los estudiantes, mostrar respeto siempre por los estudiantes, escuchar siempre a los estudiantes e incluirlos en la toma de decisiones, entre otras, y en otros estudios así se ha demostrado ${ }^{15,66}$.

En esta línea, los resultados encontrados por STUHR ${ }^{84}$, muestran que la experiencia emocional positiva de los profesores, hace que la enseñanza sea más efectiva, fomentando relaciones afectuosas y de apoyo con los estudiantes, ayudando a que alcancen los objetivos de aprendizaje deseados dentro de la clase y mejoran el conocimiento docente. Además, las buenas relaciones pueden ayudar a mejorar la participación de los estudiantes en las clases, las relaciones interpersonales entre el alumnado y los profesores, y desarrolla la autonomía en el aprendizaje del estudiante ${ }^{84,85}$.

Parafraseando a Moreno, Cervelló, Martínez y Alonso ${ }^{86}$ y a Siedentop ${ }^{69}$, para poder orientar un proceso efectivo en la asignatura de educación física, el profesorado debe de procurar por una relación calurosa y de preocupación por sus estudiantes, construir normas claras y positivas con los estudiantes, informar a los estudiantes lo que se espera de ellos, dar refuerzos frecuentes y apropiados, hacer interacciones no verbales eficaces y positivas y facilitar la solución del conflicto entre los protagonistas de la clase y el reconocimiento de los comportamientos inapropiados, permitiendo que se reconozca por cada sujeto.

Por otra parte, los estudiantes creen que un buen profesor es aquel que respeta a los estudiantes y los tratan de forma correcta; explica bien para qué sirven los ejercicios y actividades que se realizan en las clases, cuáles son sus objetivos y cómo deben de ser hechos; es justo en las pruebas y cuando evalúa; y establece junto con los estudiantes, reglas de comportamiento, lo que los estudiantes y el profesor deben de respetar. Son comentarios que concuerdan con lo que Hernández y López ${ }^{79}$ a través de su trabajo identificaron que la seguridad y la preparación que muestra el profesorado, son los rasgos más destacados por los estudiantes dentro del perfil del profesorado de educación física. En esta misma línea Cairney, Kwan, Velduizen, HaY, BraY, y FAUGHT ${ }^{87}$, mostraron cómo ese interés se manifiesta por un ambiente positivo para la adherencia del alumnado, y coincide con otros estudios en este sentido ${ }^{88-91}$.

Teniendo presente los testimonios de los estudiantes sobre el conocimiento del profesorado, es necesario e importante que el profesorado de educación física transmita a los estudiantes los conocimientos que lo ayuden a comprender por qué y para qué de lo que realizan en la asignatura, llevándolo hacia un cambio de sus creencias, percepciones y actitudes hacia la educación física. El profesorado debe de propender por la reflexión sobre las características, valores y finalidades de lo que sucede y se desarrolla en las clases de educación física en la escuela.

Sin embargo, se debe de tener presente que conocer los contenidos de enseñanza por parte del profesorado no es la verdadera esencia pedagógica a la hora de enseñar, sino que este aspecto se debe de relacionarse e integrar con el resto del proceso de enseñanza y aprendizaje. Despertar el interés y la motivación de los estudiantes hacia la asignatura de educación física, requiere de un profesorado entusiasmado, que viva y sienta la asignatura; así se transmitirá ese entusiasmo a los estudiantes y el deseo de aprender en la asignatura. Es decir, se debe integrar con los objetivos, métodos, evaluación, el contexto, las características de los estudiantes, etc. ${ }^{92}$.

A manera de conclusión se puede decir que, las percepciones del alumnado sobre sus docentes es un tema interesante, abundar en ello lleva consigo mantener una actualidad que no debería cejar. En nuestro estudio, hemos intentado dar a entender que determinadas conductas del docente son percibidas como de ayuda al alumnado, mejoran la participación en las clases de EF, y significan una mejor comprensión sobre lo que se trata de enseñar en las clases.

La elección del estudio de caso con la utilización de diferentes instrumentos, tanto cuantitativo como es el cuestionario, como cualitativo, con grupo focal y observación, nos permite contrastar y profundizar en las percepciones y creencias del alumnado con más garantías que usando instrumentos únicos. Este enlace de instrumentos permite matizar lo que se 
comprueba por un uso único. En nuestro estudio, hemos intentado dar a entender que determinadas conductas como el trato que recibe el alumnado del profesorado, el acceso al profesorado, la disciplina y manejo de conductas disruptivas y sobre los conocimientos del profesorado. El trato que percibe el alumnado (cuando reclama que sea tratado con respeto), el mantenimiento de las disciplina en la clase (para evitar alteraciones del buen clima y ambiente de clase), así como la necesidad de que muestre unos conocimientos correctos sobre lo que tiene que enseñar (demuestra dominio de lo que tiene que enseńar y se esfuerza para que el alumnado aprenda), son resultados que destacamos de nuestro estudio, pues se han obtenido con una mezcla metodológica que permite profundizar y aclarar con más énfasis estos aspectos.

Según el objetivo de nuestro estudio con el que pretendemos analizar e interpretar las creencias y percepciones del alumnado hacia la asignatura de educación física y el profesorado, podemos decir que la percepción general del alumnado sobre sus profesores es un tema interesante, abundar en ello lleva consigo mantener una actualidad que no debería cejar.

Creemos que estos resultados pueden ayudar al profesorado para mejorar su docencia. La aportación de este estudio servirá para mejorar la calidad de la enseñanza en las clases de educación física. Por otro lado, es necesario e interesante que futuras investigaciones e investigadores, construyan relaciones de complementariedad entre los dos enfoques cualitativo y cuantitativo, ayudando mejor a la compresión de la realidad. De la misma manera, convendrá para estudios futuros contrastar la perspectiva del alumnado y la del profesorado, con el fin de comprobar qué diferencias y qué acuerdos tienen los dos puntos de vista sobre un mismo proceso de interacción en las clases de EF.

\begin{abstract}
What do students think about their physical education teachers?

Research about the students' thinking presented in this work, is based on the active and essential role in their own learning process. This kind of research shows that what students create, think and feel, can affect the way they understand the contents taught, the value they may have for their lives, their interest in the subject and learning. Thus, the aim of this study was to interpret and analyze the beliefs and perceptions of the students about their physical education teachers. This was a qualitative research. Its design is case study, it was used questionary, focus groups and observation as the data collection strategies in it. The study was conducted in a school in Medellin-Colombia with participation of three teachers and 103 students of last year of high education. As conclusion, the students believe that a good teacher is one who respects and treats all equally, without discrimination, explains appropriately why choose topics and activities, explains his purpose, he is fair with the tests and when evaluating. Students have an imagen of teachers between two directions: a) teacher like a friend and guide, who offer positive aspects to their education; b) undemanding teacher, who doesn't have authority and leadership.
\end{abstract}

KEYwords: Case Study; Student Thinking; Faculty; Teaching-Learning Process.

\title{
Referencias
}

1. Sicilia Camacho Á, Fernández-Balboa J-M. Capítulo I. Introducción. La otra cara de la enseñanza: la Educación Física desde una perspectiva crítica. In: Sicilia Camacho Á, Fernández-Balboa J-M, editores. La otra cara de la enseñanza en la educación física. Barcelona: Inde; 2005. p. 13-20.

2. Morin E. La mente bien ordenada. Barcelona: Barral; Seix; 2000.

3. Coll C. Elementos para el análisis de la práctica educativa. In: Genovard Rosello C, Beltrán Llera J, Rivas Martinez F, editores. Psicología de la instrucción III: nuevas perspectivas. Madrid: Síntesis; 1995. p. 89-102. 
4. Entwistle N. La comprensión del aprendizaje en el aula. Barcelona: Paidós; 1998.

5. Mayer R. Psicología de la Educación: la enseńanza del aprendizaje significativo. Madrid: Pearson; 2004. Vol. 2.

6. Lee A. Contributions of research on student thinking in physical education. J Teach Phys Educ. 1997;16(3):262-77.

7. Wittrock M. Procesos de pensamiento de los alumnos. In: Wittrock MC, compilador. La investigación de la enseñanza, III: profesores y alumnos. Barcelona: Paidós; 1990. p. 544-85.

8. Silverman S. Trends and analysis of research on teaching in doctoral programs. J Teach Phys Educ. 1987;7(1):61-70.

9. Silverman S, Manson M. Research on teaching in physical education doctoral dissertations: a detailed investigation of focus , method, and analysis. J Teach Phys Educ. 2003;22(3):280-97.

10. Chatoupis C, Vagenas G. An analysis of published process-product research on physical education teaching methods. Int J Appl Sports Sci. 2011;23(1):271-89.

11. Torres J. La práctica reflexiva y la comprensión de lo que acontece en las aulas. In: Jackson PW. La vida en las aulas. Madrid: Morata; 2010. p. 11-26.

12. Palma A. Educação física, corpo e saúde: uma reflexão sobre outros "modos de olhar". Rev Bras Ciênc Esporte. 2001;22(2):23-39.

13. Posner C. Enseñanza efectiva. Una revisión de la bibliografía más reciente en los países europeos y anglosajones. Rev Mex Investig Educ. 2004;9(21):277-318.

14. Clark C, Peterson P. Procesos de pensamiento de los docentes. In: Wittrock MC. La investigación de la enseñanza, III: profesores y alumnos. Barcelona: Paidós; 1990. p. 443-539.

15. Piéron M. Para una enseñanza eficaz de las actividades físico-deportivas. Barcelona: Inde; 1999.

16. Sicilia Á. La investigación sobre el pensamiento del alumnado. Una revisión desde la Educación Física. Rev Educ. 2003;331:577-613.

17. Shulman L. Paradigmas y programas de investigación en el estudio de la enseñanza. Una perspectiva contemporánea. In: Wittrock MC. La investigación de la enseñanza, I: enfoques, teorías y métodos. Barcelona: Paidós; 1989. p. 9-91.

18. Pérez Gomez A. Paradigmas contemporáneos de investigación didáctica. In: Gimeno Sacristan J, Perez Gomez A. La enseñanza: su teoría y su práctica. Madrid: Akai; 2008. p. 95-138.

19. Harnischfeger A, Wiley DE. Teaching-learning processes in elementary school: a synoptic view. Curric Inq. 1976;6(1):5-43.

20. Gil Madrona P, Silva SAPS, Romo Perez V, Miranda MLJ. Actitudes de estudiantes de primaria en relación a las clases de Educación Física. Rev Bras Educ Fís Esporte (São Paulo). 2015;29(1):127-37.

21. Bruner J. Acción, pensamiento y lenguaje. Madrid: Alianza; 1984.

22. Aubert A, Flecha M, García C, Flecha R, Racionero S. Aprendizaje dialógico en la sociedad de la información. Barcelona: Hipatia; 2008.

23. Carreiro da Costa F. Issues in research on teaching in Physical Education. In: Seghers J, Vangrunderbeek K. Physical Education Research. What is the Evidence? Leuven: Acco; 2008. p. 11-25.

24. Carreiro da Costa F. Condiçóes e factores de ensino-aprendizagem e condutas motoras significativas: uma análise a partir da investigação realizada em Portugal. 6o Congreso Galego de Educación Física; 1996; Coruña, ES. Coruña: Universidade da Coruña; 1998. p. 427-54.

25. Carreiro da Costa F. Condiçóes e factores de ensino-aprendizagem e condutas motoras significativas: uma análise a partir da investigação realizada em Portugal. Bol Soc Port Educ Física. 1996;14:7-32.

26. Pereira P, Carreiro da Costa F, Diniz J. O pensamento e do aluno em Física. 6o Congreso Galego de Educación Física; 1996; Coruña: Universidade da Coruña; 1998.

27. Henrique J. Processos mediadores do professor e do aluno: uma abordagem quali-quantitativa do pensamento do professor, da interação pedagógica e das percepções pessoais do aluno na disciplina de Educação Física [dissertação]. Lisboa (LI): Universidade Técnica de Lisboa, Faculdade de Motricidade Humana; 2004.

28. Piéron M. Para una enseñanza eficaz de las actividades físico-deportivas. Barcelona: Inde; 2005.

29. Dyson B. Students' perspectives of physical education. In: Kirk D, Macdonald D, O’Sullivan M, editores. The handbook of physical education. Thousand Oaks: Sage; 2006. p. 326-46.

30. Hernández Álvares JL, Velázquez Buendía R, Garoz I. La percepción de eficacia motriz del alumnado y su relación con la actividad física y los comportamientos docentes. In: Hernández Álvares JL, Velázquez Buendía R, coordinadores. La educación física a estudio: el profesorado, el alumnado y los procesos de enseñanza: 281. Barcelona: Graó; 2010. p. $143-63$.

31. Hernández Álvares JL, Álvarez MJ. Cómo perciben los alumnos y las alumnas el comportamiento instructivo del profesorado. In: Hernández Álvares JL, Velázquez Buendía R, coordinadores. La educación física a estudio: el profesorado, el alumnado y los procesos de enseñanza: 281. Barcelona: Graó; 2010. p. 95-118. 
32. Murcia Peña N, Jaramillo L. Imaginarios del joven colombiano ante la clase de educación física. Rev Latinoam Cienc Soc Nińez Juv. 2005;3(2):2-28.

33. Moreno Murcia JA, Hellín Gómez P. Pensamiento del alumno sobre la educación física según la edad. Apunts. 2006;85(3):28-35.

34. O’Donovan T, Kirk D. Reconceptualizing student motivation in physical education: An examination of what resources are valued by pre-adolescent girls in contemporary society. Eur Phys Educ Rev. 2008;4(1):71-91. doi: 10.1177/1356336X07085710.

35. Rikard GL, Banville D. High school student attitudes about physical education. Sport Educ Soc. 2006;11(4):385-400. doi: 10.1080/13573320600924882.

36. Zeng H, Hipscher M, Leung R. Attitudes of high school students toward physical education and sport activity preferences. J Soc Sci. 2011;7(4):529-37. doi: 10.3844/jssp.2011.529.537.

37. Bernstein E, Phillips S, Silverman S. Attitudes and perceptions of middle school students toward competitive activities in Physical Education. J Teach Phys Educ. 2011;30(1):69-83.

38. Constantinou P, Manson M, Silverman S. Female students' perceptions about gender-role stereotypes and their influence on attitude toward Physical Education. Phys Educ. 2009;66(2):85-96.

39. Tannehill D, Zakrajsek D. Student attitudes towards Physical Education: a multicultural study. J Teach Phys Educ. 1993;13(1):78-84.

40. Gonçalves C. Relações entre características e crenças dos alunos e os seus comportamentos nas aulas de educação física [tese]. Lisboa (LI): Universidade Técnica de Lisboa; 1998.

41. Moreno Murcia JA, Cervelló E. Pensamiento del alumno hacia la educación física: su relación con la práctica deportiva y el carácter del educador. Enseñanza. 2003;21:345-62.

42. Zeng HZ, Hipscher M, Leung RW. Attitudes of high school students toward physical education and sport activity preferences. J Soc Sci. 2011;7(4):529-37.

43. Cuevas R, Onofre Contreras, García-Calvo T. Effects of an experimental program to improve the motivation in Physical Education of spanish students. Procedia Soc Behav Sci. 2012;47:734-8. doi: 10.1016/j.sbspro.2012.06.726.

44. Fisette JL. 'Are you listening?' Adolescent girls voice how they negotiate self-identified barriers to their success and survival in physical education. Phys Educ Sport Pedagog. 2013;18(2):184-203.

45. Cubero R. Perspectivas constructivistas. La intersección entre el significado, la interacción y el discurso. Barcelona: Graó; 2005.

46. López Fuentes R. Creencias del profesorado universitario sobre evaluación [tesis]. Granada (AN): Universidad de Granada; 2001.

47. Soto González JI. Conocimientos y creencias sobre la formación en valores y técnicas de intervención del alumnado de magisterio en Granada [tesis]. Granada (AN): Universidad de Granada; 2011.

48. Pehkonen E, Törner G. Mathematical beliefs and different aspects of their meaning. Int Rev Math Educ. 1996;28(4):101-8.

49. Escámez J, García López R, Pérez C, LLopis Blasco JA. El aprendizaje de valores y actitudes: teoría y práctica. Barcelona: Octaedro; 2007.

50. Isen AM. An influence of positive affect on decision making in complex situations: theoretical issues with practical implications. J Consum Psychol. 2001;11(2):75-85. doi: 10.1207/S15327663JCP1102_01.

51. Fredrickson BL. The broaden-and-build theory of positive emotions. Philos Trans R Soc Lond B Biol Sci. 2004;359(1449):1367-78.

52. Coller X. Estudio de casos. Madrid: CIS; 2000.

53. Merriam SB. Case study research in education: a qualitative approach. San Francisco: Jossey-Bass; 1988.

54. Stake R. Investigación con estudio de casos. Madrid: Morata; 1998.

55. Yin RK. Case study research: design and methods. Newbury Park: Sage; 1984. (Applied Social Research Methods, 5).

56. Galeano M. Estrategias de investigación social cualitativa: el giro en la mirada. Medellín: La Carreta; 2004.

57. Santos M. Hacer visible lo cotidiano. Teoría práctica de la evaluación cualitativa de los centros escolares. Madrid: Akal; 1993.

58. Hamui-Sutton A, Varela M. La técnica de grupos focales. Investig Educ Médica. 2013;2(1):55-60.

59. Taylor S, Bogdan R. Introducción a los métodos cualitativos de investigación. Barcelona: Paidós; 1992.

60. Nolasco RC. As percepçôes pessoais, crenças e valores dos alunos na disciplina de Educação Física [dissertação]. Rio de Janeiro (RJ): universidade Castelo Branco; 2007.

61. Capel S. Moving beyond physical education subject knowledge to develop knowledgeable teachers of the subject. Curric J. 2007;18(4):493-507. doi: 10.1080/09585170701687936. 
62. Capel S, Katene W. Secondary PGCE PE students' Perceptions of their subject knowledge. Eur Phys Educ Rev. 2000;6(1):46-doi: 10.1177/1356336X000061005.

63. Murcia Peña N, Jaramillo L. Imaginarios del joven colombiano ante la clase de educación física. Educación Física y Deportes [Internet]. 2005 [citado 22 jul 2019];10(83). Disponível em: http://www.efdeportes.com/ efd83/imag.htm.

64. Devís J. Educación física, deporte y currículum. Investigación y desarrollo curricular. Madrid: Visor; 2000.

65. Figueiredo Z, Silva E, Andrade N, et al. Educação física, ser professor e profissão docente em questão. Pensar a Prática. 2008;11(2):209-18.

66. Florence J. Tareas significativas en Educación Física Escolar. Barcelona: Inde; 1991.

67. Piéron M, Delmelle R. Les réactions a la prestation de l'élève. Étude dans l'enseignement de la Danse Moderne. Rev l'Éducation Phys. 1983;23(4):35-41.

68. Rivera Garcia E, Trigueros Cervantes C, Pavesio Estero M. Líneas de investigación en Educación Física Escolar. Ágora. 2003;2(3):75-88.

69. Siedentop D. Aprender a enseñar la educación física. Barcelona: Inde; 2008.

70. Tinning R, Siedentop D. The characteristics of tasks and accountability in student teaching. J Teach Phys Educ. 1985;4(4):286-99.

71. Viciana J, Cervelló E, Ramírez J, San Matías J, Requena B. Inluencia del feedback positivo y negativo en alumnos de Secundaria sobre el clima ego-tarea percibido, la valoración de la EF y la preferencia en la complejidad de las tareas de clase. Rev Mot. 2003;10:99-116.

72. Griffin P. Boys' participation styles in a middle school physical education team sports unit. J Teach Phys Educ. 1985;4:100-10.

73. Velázquez Buendía R, Hernández Álvarez JL, Garoz Puerta I, et al. Calidad de enseñanza en Educación Física y Deportiva y discurso docente: el caso de la Comunidad de Madrid. Rev Educ. 2007;344:447-67.

74. Velázquez Buendía, López Rodríguez Á. El proceso de comunicación docente-discente en educación física. In: Hernández Álvarez JL, Velázquez Buendía R, coordinadores. La educación física a estudio: el profesorado, el alumnado y los procesos de enseñanza. Barcelona: Graó; 2010. p. 37-67.

75. Monique L, Malini R. Content knowledge in teaching, an investigation into an adequate "milieu" for teaching dance: the case of Indian dance in France. Eur Phys Educ Rev. 2010;16(1):65-79. doi: 10.1177/1356336X10369198.

76. Amade-Escot C, O'sullivan M. Research on content in physical education: theoretical perspectives and current debates. Phys Educ Sport Pedagog. 2007;12(3):185-204. doi: 10.1080/17408980701610144.

77. Ayvazo S, Ward P. Pedagogical content knowledge of experienced teachers in Physical Education: functional analysis of adaptations. Res Q Exerc Sport. 2011;82(4):675-84. doi: 10.1080/02701367.2011.10599804.

78. Galeano J. Para ser Educador en el Siglo XXI, un texto de apoyo a la transformación curricular en Normales, Facultades, Escuelas e Institutos. Medellín: La Carreta; 2002.

79. Luis Hernández J, López C. Qué piensan los niños, niñas y adolescentes de la educación física y de sus profesores y profesoras. In: Luis Hernández J, Velázquez R, coordinadores. La educación física, los estilos de vida y los adolescentes: cómo son, cómo se ven, qué saben y qué opinan: 242. Barcelona: Graó; 2007. p. 143-68.

80. Batalloso JM. ¿Es posible una evaluación democrática? O sobre la necesidad de avaluar educativamente. In: Ballester M, Batalloso JM, Calatayud MA, et al. Evaluación como ayuda al aprendizaje. Barcelona: Graó; 2000. p. 45-54.

81. Ferrández Marco MC, Perandones González T, Grau Company S. El porqué de la pasión y el entusiasmo en contextos escolares. In:Caruana Vaño A, coordinador. Aplicaciones educativas de la psicología. Alicante: Hispania; 2010. p. 72-89.

82. Graham G. Physical education through students' eyes and in students' voices: implications for teachers and researchers. J Teach Phys Educ. 1995;14(4):478-82.

83. Tapia J. Motivación para el aprendizaje: la perspectiva de los alumnos. In: Rivera Otero A, María Pérez S, coordinadores. La orientación escolar en centros educativos. Madrid: Ministerio de Educación Cultura y Deporte; Secretaría General Técnica; 2005. p. 209-42.

84. Stuhr PT. Teaching with feeling: the essence of lived-positive emotionality and care among physical education teachers and their students [dissertation]. Columbus (OH): Ohio State University; 2008.

85. Martín X, Puig J. Las siete competencias básicas para educar en valores. Barcelona: Graó; 2007.

86. Moreno Murcia JA, Cervelló Gimeno E, Martínez Galindo C, Alonso Villodre N. Los comportamientos de disciplina e indisciplina en educación física. Rev Iberoam Educ. 2007;44:167-90. 
87. Cairney J, Kwan MYW, Velduizen S, Hay J, Bray SR, Faught BE. Gender, perceived competence and the enjoyment of physical education in children: A longitudinal examination. Int J Behav Nutr Phys Act. 2012;9:26. doi: 10.1186/14795868-9-26.

88. Díaz del Cueto M, Aguado Gómez R. Percepción de competencia del profesorado de educación física con experiencia sobre la tarea como recurso didáctico. Retos. 2012;22:16-8.

89. Gutiérrez M, Pilsa C. Students' attitudes towards physical education and their teachers. Rev Int Med Cienc Act Fís Deport. 2006;6:212-29.

90. Gutiérrez M, Ruiz LM, López E. Motivational climate in physical education: correlates between students' and their teachers' perceptions. Rev Psicol Deport. 2011;20(2):321-35.

91. Whitehead S, Biddle S. Adolescent girls' perceptions of physical activity: a focus group study. Eur Phys Educ Rev. 2008;14(2):243-62. doi: 10.1177/1356336X08090708.

92. Contreras Jordán OR. Las competencias del profesor de educación física. In: Contreras Jordán OR. Las competencias del profesor de educación física. Barcelona: Inde; 2012. p. 11-30.

Didier Fernando Gaviria Cortes Ciudadela Robledo bloque 45 of. 102 - 05001000 Medellín - Antioquia - COLOMBIA e-mail: didier.gaviria@udea.edu.co; gaviriadidier@yahoo.es
Recibido: 02/05/2016 $1^{\text {a }}$ Revisión: 23/09/2017 $2^{\text {a }}$ Revisión: 02/02/2018 Aprobado: 09/04/2018 